\title{
Profile of atacicept and its potential in the treatment of systemic lupus erythematosus
}

This article was published in the following Dove Press journal:

Drug Design, Development and Therapy

5 March 2015

Number of times this article has been viewed

\section{Estafania Cogollo',* \\ Marta Amaral Silva ${ }^{2, *}$ \\ David Isenberg ${ }^{3}$}

'Department of Internal Medicine, Hospital Principe de Asturias, Alcala de Henares, Madrid, Spain; ${ }^{2}$ Department of Internal Medicine, Hospital Distrital da Figueira da Foz, Coimbra, Portugal; ${ }^{3}$ Centre for Rheumatology, Department of Medicine, University College London, London, UK

*These authors are regarded as equal first authors
Correspondence: D Isenberg Room 424, The Rayne Building, University College London, 5 University Street, London WCIE 6JF, UK Email d.isenberg@ucl.ac.uk

\begin{abstract}
The importance of B cell activating factors in the generation of autoantibodies in patients with systemic lupus erythematosus (SLE) is now recognized. The two key factors, known as BAFF and APRIL, produced by a variety of cells including monocytes, dendritic cells and T cells, also help to regulate B cell maturation, function and survival. Biologic agents that block these factors have now been developed and tried out in large scale clinical trials in SLE patients. Benlysta which blocks BAFF has met some of its end points in clinical trials and is approved for use in patients with skin and joint disease who have failed conventional drugs. In contrast, clinical trials using atacicept which blocks both BAFF and APRIL have been more challenging to interpret. An early study in lupus nephritis was, mistakenly, abandoned due to serious infections thought to be linked to the biologic when in fact the dramatic fall in the immunoglobulin levels took place when the patients were given mycophenolate, prior to the introduction of the atacicept. Likewise the higher dose arm (150 mgm) of a flare prevention study was terminated prematurely when 2 deaths occurred. However, the mortality rate in this study was identical to that seen in the Benlysta studies and a post hoc analysis found a highly significant benefit for the $150 \mathrm{mgm}$ arm compared to the lower dose $(75 \mathrm{mgm})$ and placebo arms. Other trials with
\end{abstract} both Benlysta and atacicept are on-going.

Keywords: cytokines, lupus nephritis, BLyS, APRIL

\section{Introduction}

Systemic lupus erythematosus (SLE) is an autoimmune rheumatic disease in which autoantibodies target a variety of self-antigens, leading to many clinical manifestations. ${ }^{1}$ Despite great improvement in outcome during the past 50 years, many patients continue to experience significant morbidity or even die prematurely. ${ }^{2,3}$ Lupus nephritis (LN) in particular is often a severe complication of SLE whose treatment may have significant toxicity and many patients do not achieve complete remission. ${ }^{4}$ It has been anticipated that biological drugs targeting key molecules or cells will optimize the treatment of SLE. ${ }^{3,5,6}$ Given the role of B lymphocytes in the pathogenesis of SLE, rituximab, a monoclonal antibody directed to CD20, was studied in this disease. After some disappointing results, interest focused on targeting B-cell activating factors like the B-lymphocyte stimulator (BLyS). Belimumab (monoclonal antibody to BLyS) was approved for the treatment of SLE. Atacicept is a fusion protein that blocks not only BLyS but also the proliferation-inducing ligand (APRIL), another B-cell activating factor. It seems reasonable to assume that neutralizing both BLyS and APRIL would have different biological consequences and be more efficacious than neutralization of BLyS alone. ${ }^{1,3,7,8}$ This article reviews the main published data about atacicept. 


\section{Background}

\section{Targeting B cells: the role of rituximab}

$B$ cells have been shown to be important in the pathogenesis of SLE and other autoimmune diseases such as rheumatoid arthritis (RA), multiple sclerosis (MS), and neuromyelitis optica. ${ }^{1,9,10}$ They play a key role mediating immune responses through autoantibody production and autoantigen presentation, interactions with $\mathrm{T}$ and dendritic cells and cytokine production. ${ }^{1,3}$ Autoantibodies directed against a large number of self-antigens in the nucleus, cytoplasm, and cell membranes develop and bind to their targets, leading to the formation of immune complexes. These complexes activate complement resulting in a cascade of inflammatory reactions. ${ }^{1}$ It was hoped that successfully targeting B cells would improve clinical outcome with fewer side effects than conventional therapies. Several strategies have been employed to target B-lymphocytes. ${ }^{1,3}$

Interest initially focused on removing CD20 positive B-lymphocytes in patients with SLE using rituximab, a chimeric monoclonal antibody directed to CD20. ${ }^{2}$ Success was initially reported in case series of patients with active/ refractory SLE. ${ }^{11}$ However, two double-blind controlled trials - EXPLORER and LUNAR, which evaluated rituximab in patients with non-renal lupus and with LN, respectively, showed disappointing results. ${ }^{12,13}$ These may reflect problems with trial design, notably the intensive use of concomitant glucocorticoids and immunosuppressives.

\section{BLyS and APRIL: from belimumab to atacicept}

The tumor necrosis factor (TNF) ligand superfamily has two related members that regulate B-cell maturation, function and survival: BLyS, also known as B-cell activating factor belonging to the TNF family (BAFF), and APRIL. ${ }^{9}$ These molecules are produced by monocytes, dendritic cells, macrophages, and T cells. ${ }^{14} \mathrm{BLyS}$ is a type II transmembrane protein with 285 amino acids. When surface BLyS is cleaved by a furin protease, a soluble, biologically active $17 \mathrm{KDa}$ molecule is released and binds to three receptors on the surface of $\mathrm{B}$ cells: B-cell maturation antigen (BCMA), transmembrane activator and calcium-modulating ligand interactor (TACI) and BR3 (also known as BAFFR). ${ }^{7,14}$ APRIL is synthetized as a type II transmembrane protein that is intracellularly proteolytically cleaved before the secretion of the biologically active form. APRIL binds to two BLyS receptors (BCMA and TACI) and also to the heparin sulfate proteoglycans. ${ }^{14}$ Both BLyS and APRIL enhance the survival of human memory B cells and thus promote their differentiation into plasma cells. This is likely to be mediated by their interaction with BCMA. ${ }^{14}$ Furthermore, it was suggested that the APRIL/BLyS/TACI interactions play an important role in immunoglobulin (Ig)A class switch recombination. ${ }^{15}$

Studies have shown that the regulation of the pre-immune B cell pool depends upon BLyS/BR3 signaling and that mature primary B cells require BLyS for survival. Elevated BLyS levels allow B cells to pass the transitional checkpoint and become established in the mature follicular and marginal zone B cell pools. In contrast, reducing available BLyS may lead to a more rigorous selection and reduce mature $\mathrm{B}$ cell lifespan thus reducing the number of mature B cells. ${ }^{16}$ Analysis of BLyS deficient mice with lower numbers of mature B cells and of BLyS transgenic (Tg) mice developing severe B-cell hyperplasia support the notion of an important role for BLyS in B-cell maintenance. ${ }^{17}$ Repeated administration of BLyS to mice results in B cell expansion and polyclonal hypergammaglobulinemia. ${ }^{18}$ Unlike soluble BLyS, the role of membrane BLyS remains uncertain. ${ }^{7}$

APRIL upregulates co-stimulatory molecules on B cells enhancing their antigen presentation function. ${ }^{19}$ One in vitro study reported that APRIL co-stimulates mouse T-cell proliferation. ${ }^{20}$ In addition, APRIL Tg mice have an increased T-cell survival. ${ }^{14}$ An increase in spleen weight and in the proportion of B cells in the spleen was observed after the administration of recombinant soluble APRIL to mice. ${ }^{20}$ Other authors reported an increase in the percentage of $\mathrm{B}$ cells in the peripheral lymph nodes in mice that overexpress a human APRIL transgene. ${ }^{21}$ An increase in IgM (in response to $\mathrm{T}$-dependent and $\mathrm{T}$-independent antigens) and in $\mathrm{IgG}$ (in response to T-independent antigens) was reported in APRIL Tg mice. ${ }^{14,22}$

Interestingly, BLyS and APRIL also promote the survival of regulatory B cells which down-modulate immune responses. It remains unknown why the final effect of these cytokines in a given host is variably pro- or antiautoimmunity. ${ }^{8}$

Signals from BLyS and APRIL induce proliferation of plasma cells and thus increased Ig production. Some of the antibodies produced are likely to be directed to selfantigens. ${ }^{23,24}$ Studies have demonstrated that constitutive overexpression of BLyS often leads to the development of SLE-like features (including high levels of circulating autoantibodies and renal Ig deposits) in BLyS-Tg non-autoimmune prone mice and accelerates target-organ (kidney) pathology in autoimmune prone mice. ${ }^{25-28}$ Although mice with a lifelong absence of BLyS were not protected from serological autoimmunity and renal pathology, they were 
largely spared from clinically overt disease (severe proteinuria and premature death) ${ }^{23}$ Conversely, the role of APRIL in these pathologies is controversial. ${ }^{8,29}$ Two studies generated APRIL deficient mice and found conflicting results. In one of them, no effects on $\mathrm{T}$ and $\mathrm{B}$ cells' development and in vitro function or in vivo humoral responses were observed. ${ }^{30}$ In the other group, although APRIL knockout mice had a normal $\mathrm{T}$ and $\mathrm{B}$ cell development and proliferation in vitro, they had an increased number of effector memory $\mathrm{T}$ cells, enlarged germinal centers and an increased IgG response to T-dependent antigens. ${ }^{31}$ These disparate results can be explained by the different mouse genetic background. In a study in which mice were genetically rendered APRIL deficient, the authors concluded that APRIL is not essential for the development of full blown SLE in mice and may play a protective role. ${ }^{32}$ In another trial, APRIL Tg mice did not display signs of autoimmunity. ${ }^{17}$ These results highlight the need for a study with an agent that selectively blocks APRIL without also blocking BLyS.

Increased levels of APRIL, BLyS homotrimers, and/or BLyS/APRIL heterotrimers have been observed in humans with various autoimmune disorders, including SLE, as well as in the arthritic joints of patients with inflammatory arthritis. ${ }^{1,3,9,14,16,29,33}$ In addition, high serum levels of BLyS and APRIL have been described in patients with MS and chronic lymphocytic leukemia (CLL). ${ }^{34,35}$ It is known that CLL patients with a low percentage of leukemic cells expressing BLyS or APRIL have a significantly longer overall survival than patients with BLyS- or APRIL-positive leukemic cells. ${ }^{35}$ In patients with SLE, the concentration of these cytokines positively correlates with disease severity and serological markers such as anti-double-stranded DNA antibody levels, suggesting that BLyS and APRIL play a significant role in the pathogenesis of this disease. ${ }^{5,36}$ Conflicting results were reported in another study where serum APRIL levels were reported to be inversely correlated with serum anti-dsDNA titers and clinical disease activity, suggesting that APRIL might play a protective role in SLE patients. ${ }^{37}$ Additional studies are needed to clarify the role of APRIL is this disease.

Reviewing the unsatisfactory results of some rituximab trials and the biological effects of BLyS, it is reasonable to ask whether the indirect targeting of $\mathrm{B}$ cells via a survival factor might be preferable to targeting the B cells directly. ${ }^{7}$ Studies in mice strongly suggest that some autoreactive $\mathrm{B}$ cells have a greater dependency on BLyS for their survival than do non-autoreactive B cells and, thus, blocking BLyS could preferentially eliminate pathogenic B cells while sparing protective B cells. ${ }^{7}$ Furthermore, mice that overexpress BLyS inflammatory responses are deviated toward a T helper 1 cell profile and away from a Th2 cell profile via a B-cell-independent pathway. ${ }^{38}$ Moreover, through direct effects on $\mathrm{T}$ cells, BLyS promotes in vitro generation of Th17 cells. ${ }^{39}$ Thus, targeting BLyS may not only target pathogenic B cells but also potentially, pathogenic Th1 and/ or Th17 cells. ${ }^{7}$

Belimumab was developed as an alternative approach to B-cell-directed therapy. ${ }^{3}$ It is a human $\operatorname{IgG} 1 \lambda$ monoclonal antibody that binds soluble human BLyS and inhibits its biologic activities. ${ }^{3,7}$ Belimumab was designed to avoid direct killing of $\mathrm{B}$ cells, by preventing antibody-dependent cell cytotoxicity, and the complement activation cascade. ${ }^{40}$ A Phase II trial was performed to evaluate efficacy and safety of belimumab in patients with active SLE with a SELENASLEDAI (Safety of Estrogen in Lupus Erythematosus National Assessment - Systemic Lupus erythematosus Disease activity Index) score of $\geq 4 .{ }^{41}$ Patients were randomized to belimumab plus standard-of-care or placebo and followed up for 52 weeks. Co-primary efficacy endpoints were the percentage change in SELENA-SLEDAI from baseline to week 24 and time to first flare. Although belimumab was biologically active, as demonstrated by reductions in some B cell populations, Ig levels and anti-dsDNA antibodies, there was no significant reduction of SLE disease activity or flares in patients treated with this drug compared to placebo. In a post hoc analysis of the Phase II trial, belimumab had significant, albeit modest benefit in patients with SLE. ${ }^{42}$ BLISS (Belimumab in subjects with Systemic Lupus Erythematosus) 52 and BLISS 76 were Phase III double-blind, randomized, controlled trials performed in patients with serologically active SLE with SELENA-SLEDAI scores of $\geq 6 .{ }^{43,44}$ Patients had primarily cutaneous and musculoskeletal manifestations of SLE. The primary endpoint was the SLE responder index (SRI) response rate at week 52 (an SRI response was defined as a $\geq 4$ point reduction in SELENA-SLEDAI score, no new British Isles Lupus Assessment Group A organ domain score and no more than one new British Isles Lupus Assessment Group B score, and no worsening in physician's global assessment score versus baseline). In both studies, patients treated with belimumab plus standard-of-care had significantly improved SRI response rates compared with placebo. Pooled analyses of these trials confirmed a modest, but statistically significant, therapeutic effect of belimumab in SLE. ${ }^{45}$ Nevertheless, the results were disappointing because overall the degree of improvements in those patients given belimumab was not impressively different from those given 
placebo. These results might be explained by the fact that they were conducted in different patient populations, with different therapies at baseline. Furthermore, the National Institute for Clinical Excellence in the UK, considered that the drug was not cost effective. A post hoc analysis of the Phase III trials was performed to evaluate if belimumab would offer additional benefit to patients with renal involvement receiving mycophenolate mofetil (MMF) at baseline. ${ }^{46}$ Belimumab promoted renal improvement, although the differences between groups in most renal outcomes were not significant. Of note, this analysis included a small number of patients and those with severe active LN were excluded. Furthermore, a pooled multivariate analysis of the Phase III trials showed that the therapeutic effect of belimumab was especially obvious in the more serologically active patients with high anti-dsDNA antibodies and low C3 levels. ${ }^{47}$ Despite its mixed results, the drug has been approved by the Food and Drug Administration and the European Medicines Agency for use in SLE.

Belimumab is able to bind to soluble, but not membrane BAFF, thus the only mechanism of action is a reduction of signaling through the BAFF receptors by removing soluble BAFF in circulation. As belimumab does not bind to APRIL, signaling through BAFF receptors is only partially blocked. ${ }^{40}$ It is possible that APRIL may rescue some B cells from the anti-CD20 effects of rituximab and BAFF-neutralizing effects of belimumab. ${ }^{8}$

\section{Atacicept}

Atacicept is a fully human recombinant fusion protein containing the BLyS/APRIL-binding extracellular portion of the TACI receptor fused to a slightly modified Fc portion of human IgG1. ${ }^{3}$ Unlike belimumab, this agent neutralizes both BLyS and APRIL, and prevents them from binding to their receptors on lymphocytes. ${ }^{1,3-5,7-9,16,40,48}$ Blockade of both cytokines indirectly affects mature B and plasma cells by inhibiting B cell survival after the T1 transitional stage, while sparing B-cell progenitors and memory B-cells. . $^{1,3,9,14}$ Levels of serum IgG, IgM, and IgA and numbers of mature and total circulating $\mathrm{B}$ cells are reduced by atacicept. ${ }^{4,9}$ Hence, in theory atacicept should be a viable and effective therapeutic option. ${ }^{1,7}$

In order to evaluate its efficacy and safety, atacicept has been studied in animal models and in patients with RA, MS, hematological conditions, and SLE.

\section{Preclinical studies}

Immunological differences were observed between SLE-prone mice in which both BLyS and APRIL were eliminated or neutralized and mice in which only BLyS was eliminated or neutralized. Greater immunosuppression was observed in the former. ${ }^{32,49} \mathrm{~B}-1$ cells are a subset of mature long-lived B cells. ${ }^{50}$ In a study by Gross et al B-1 cells were present in normal levels in mice in which only BLyS was neutralized, but were absent in TACI Ig Tg or treated mice. TACI Ig can neutralize heterotrimers of BLyS and APRIL. This suggests that both BLyS and APRIL are needed for the development and survival of the B-1 cell population. ${ }^{50}$ In animal models of RA, neutralization of both BLyS and APRIL was shown to reduce disease activity. ${ }^{50,51}$

A reversible sub-total depletion of $B$ cells and reduction in circulating Ig (especially IgM) levels were documented in preclinical studies with atacicept in mice and cynomolgus monkeys. The only evident systemic toxicity was transient elevation in liver transaminases (without any histological changes in the livers). ${ }^{52}$ Delays in the development of proteinuria and increased survival were observed in autoimmuneprone lupus mice treated with atacicept. ${ }^{25}$ Atacicept was also effective in the animal model of MS. ${ }^{53}$ In a rat model of optic neuritis, intraperitoneal treatment with 100 or $200 \mu \mathrm{g}$ of atacicept resulted in increased apoptosis of retinal ganglion cells. The extent of inflammation, demyelination, and axonal loss of the optic nerve was more pronounced with this dose level compared to lower doses. ${ }^{54}$

\section{Clinical studies in healthy volunteers, RA, MS, and hematological malignancies}

In a Phase 1 study healthy volunteers were given atacicept or placebo. ${ }^{33}$ The 70,210 , and $630 \mathrm{mg}$ doses demonstrated a dose-dependent biological effect on IgM levels, with no treatment-related effects on IgG levels or lymphocyte subpopulations. Subsequent human trials with atacicept have demonstrated a reduction in total serum Ig levels.

Several studies were performed to determine the efficacy of atacicept in the treatment of RA. A Phase Ib doseescalating study was undertaken in 73 rheumatoid factor (RF)-positive patients with active, moderate-to-severe RA. ${ }^{55}$ Atacicept or placebo were given as single (70, 210 or $630 \mathrm{mg}$ ) or repeated doses (three doses of 70 or $210 \mathrm{mg}$, or seven doses of $420 \mathrm{mg}$ ) every fortnight. Median IgG, IgA, and IgM values were reduced by $21 \%$, IgA $37 \%$ and $54 \%$ respectively compared to baseline. ${ }^{55}$ This is about double the reduction seen in belimumab treated patients (in a Phase II study, reductions of $10 \%, 14 \%$, and $29 \%$ were observed, respectively, in the serum concentration of $\operatorname{IgG}, \operatorname{IgA}$, and $\operatorname{IgM}$, after belimumab, compared with a reduction inferior to $5 \%$ in the placebo 
group). ${ }^{41}$ Interestingly, the highest dose of atacicept (seven doses of $420 \mathrm{mg}$ ) also reduced RF and anti-cyclic citrullinated peptide antibody levels. After an initial transient increase in the B-cell count (especially memory B cells), the number of B cells (especially mature B cells) decreased below the pre-dosing levels. Patients treated with the maximum dose of atacicept had a mean disease activity score 28 that fell from 6.4 at baseline to 5.1 on day 85 and persisted after treatment cessation. During the 3 months of atacicept treatment, $32 \%$ of patients achieved the American College of Rheumatology criteria for $20 \%$ improvement in disease severity (ACR20), with two patients achieving an ACR70 response. ${ }^{55}$

Two Phase II trials focused on efficacy, safety, and biological activity of atacicept in patients with active, moderateto-severe RA. The primary endpoint was a $20 \%$ improvement in disease severity according to the ACR criteria, assessed using the C-reactive protein level (ACR20-CRP), at week 26. In AUGUST I, 256 patients with RA in whom TNF antagonist therapy had failed, were given three different doses of atacicept $(25,75$ or $150 \mathrm{mg})$ or placebo, twice weekly for 4 weeks, then weekly for 21 weeks, with a 13 week treatment-free follow-up period (until week 38). ${ }^{56}$ In those receiving atacicept, IgM, IgA, and IgG levels decreased, in a dose-dependent manner, from the first dose to week 26. RF levels were also reduced at week 26 . Both effects on total Ig and RF were reversible after atacicept was discontinued. There were no statistically significant reductions of anti-cyclic citrullinated peptide titers. In AUGUST II, 311 TNF antagonist-naïve patients with RA in whom the response to methotrexate treatment was inadequate received either placebo, atacicept $150 \mathrm{mg}$ weekly, with or without a 4 week loading period (twice weekly dosing), or open-label adalimumab $40 \mathrm{mg}$ every other week, for 25 weeks. ${ }^{57}$ As in the former study, Ig and RF levels decreased in patients randomized to atacicept, whereas in the group of patients given adalimumab, a slight, non-statistically significant increase in the three Ig classes and RF levels was observed. Like the Phase Ib study, there was a reduction of absolute levels of mature B cells and plasma cells from baseline to week 26 in the atacicept treated groups. During the course of atacicept treatment, the number of memory B-cells transiently increased.

AUGUST I and AUGUST II failed to reach the primary endpoint. In AUGUST I, atacicept had no significant effect on the ACR20-CRP, ACR50-CRP or ACR70-CRP response rates. ${ }^{56}$ In AUGUST II the ACR50-CRP showed significantly greater response rates in the atacicept groups, although lower than in the adalimumab group, as compared with placebo. ${ }^{57}$ Nevertheless, the dose-dependent reduction of Ig and RF levels as well as the decrease in circulating levels of mature B and plasma cells, demonstrated the biological activity of atacicept.

A recent randomized, placebo-controlled, double-blind, Phase 2 trial showed an increased rate of relapse with atacicept treatment in patients with relapsing-remitting MS. This increase in clinical disease activity occurred in parallel with reductions in serum Ig concentrations and mature B-cell counts as reported in previous atacicept studies. ${ }^{34}$

Atacicept was also investigated in hematological conditions resulting from clonal proliferation of lymphocytes and plasma cells. A Phase $1 \mathrm{~b}$ clinical trial to investigate the tolerability and biological activity of escalating doses of intravenously administered atacicept in patients with refractory or relapsed CLL was performed. The results showed that patients treated with high dose atacicept $(27 \mathrm{mg} / \mathrm{kg})$ experienced stable disease or partial response. In contrast, progressive disease was observed in patients treated with low dose levels ( 1 and $4 \mathrm{mg} / \mathrm{kg}$ ). ${ }^{35} \mathrm{In}$ studies performed in patients with relapsed or refractory B-cell non-Hodgkin lymphomas, multiple myeloma and Waldenstrom's macroglobulinemia, atacicept was well tolerated and had biological activity with a decrease in all polyclonal Ig isotypes and a substantial decline in circulating Ig-producing cells. However, tumor responses were not observed. ${ }^{58,59}$

\section{Atacicept in SLE}

Atacicept has been studied in patients with SLE. A Phase Ib double-blind study in 49 patients with SLE who were randomized to either single or repeated doses of atacicept or placebo included patients who had mild to moderate SLE, but had not used MMF, cyclophosphamide, azathioprine, or methotrexate within 8 weeks of enrollment. ${ }^{60}$ Dosing regimens ranged from 0.3 to $9 \mathrm{mg} / \mathrm{kg}$, including single and multiple doses. After an initial increase, a dose response reduction in B lymphocytes was observed in all atacicept groups. No reduction in T cells, monocytes, or natural killer cells was observed. There was a reduction in Ig levels, which was more evident in IgM levels although IgG and IgA levels also declined. Both Ig and B-cell responses were greatest in the repeated dosing regimens and were maintained at day 64 suggesting a lasting effect of atacicept. Favorable safety and tolerability profiles were demonstrated. In another Phase $\mathrm{Ib}$ study patients with mild-to-moderate SLE were randomized to receive atacicept single dose: 3,9 or $18 \mathrm{mg} / \mathrm{kg}$; or multiple dose: $2 \times 9 \mathrm{mg} / \mathrm{kg}$; or placebo. ${ }^{61}$ Patients were assessed for 6 weeks, except those in the $2 \times 9 \mathrm{mg} / \mathrm{kg}$ cohort who were followed for 9 weeks. The biological activity of atacicept 
was demonstrated by a reduction of B-cells and Ig levels. IgM levels showed the greatest declines with treatment, followed by IgA and IgG. Atacicept was well tolerated, both systemically and locally.

A randomized Phase II/III trial of atacicept was designed to assess whether atacicept could prevent flares in patients with active lupus treated with a course of corticosteroids (CS). ${ }^{3}$ Patients were randomized to atacicept $75 \mathrm{mg}$ or atacicept $150 \mathrm{mg}$ administered subcutaneously, or placebo twice weekly for 4 weeks, then weekly for 48 weeks. Two fatal infections occurred in the $150 \mathrm{mg}$ atacicept arm, leading to premature termination of this group. Primary endpoint analysis was thus only performed in the $75 \mathrm{mg}$ and the placebo arm. There was no significant difference in the adjudicated flare rates in the placebo and the $75 \mathrm{mg}$ atacicept arm (flare rates of $58 \%$ and $54 \%$, respectively; odds ratio [OR] 1.15 [0.73 to 1.80$], P=0.543$ ). Post hoc analysis of the primary endpoint suggested a beneficial effect in the atacicept 150 $\mathrm{mg}$ regimen with a significant reduction in the proportion of patients with flares compared with placebo (flare rate $37 \%$ and 54\%, respectively; OR 0.48 [0.30 to 0.77], $P=0.002$ ). Flare rates were analyzed among patients who completed treatment prior to discontinuation of the $150 \mathrm{mg}$ arm. Atacicept $75 \mathrm{mg}$ had no significant benefit versus placebo (flare rate $58 \%$ and $60 \%$, respectively; OR 0.89 [ 0.48 to 1.67 ], $P=0.724$ ), but there was a significant reduction in the flare rate in the $150 \mathrm{mg}$ arm compared to placebo ( $43 \%$ and $60 \%$, respectively; OR 0.49 [ 0.26 to 0.92 ], $P=0.027$ ). The high-dose treatment was also associated with a significantly delayed time to first flare compared with placebo (hazard ratio 0.56 [0.36 to 0.87 ], $P=0.009)$. There was a dose dependent decrease in the proportion of subjects who had at least one increase in CS dose to $\geq 20 \mathrm{mg} /$ day, which suggests that atacicept may have steroid-sparing benefit. Both 75 and $150 \mathrm{mg}$ doses induced a similar reduction in anti-dsDNA antibodies. Atacicept was associated with reductions in B-cells, plasma cells and total Ig levels, and increases in $\mathrm{C} 3$ and $\mathrm{C} 4$ levels. Although the beneficial effect on $\mathrm{C} 3$ and $\mathrm{C} 4$ was more evident in those treated with atacicept $150 \mathrm{mg}$, it remains unclear why the clinical response appeared at 150 but not $75 \mathrm{mg}$ of atacicept.

The atacicept $75 \mathrm{mg}$ arm failed to reach the primary endpoint of reducing the number of SLE patients who had a new flare. Results with the dose of $150 \mathrm{mg}$ were encouraging and it is very probable that the higher dose of atacicept was effective. However, safety concerns about the risk of infection were raised. Nevertheless, as the frequency of flares may have a significant impact in the clinical outcome, it is worth investigating strategies that might reduce the flare rate.
It has been suggested that APRIL could be a potential biomarker for predicting hard-to-treat cases of LN, and the use of APRIL antagonists such as atacicept has been proposed for the treatment of LN patients with high APRIL serum levels. ${ }^{62}$

A Phase II/III randomized, double-blind, placebocontrolled clinical trial was initiated to evaluate the efficacy and safety of atacicept in patients with active LN who had recently started immunosuppressive therapy with CS and MMF. ${ }^{4}$ An unexpected decline in serum $\operatorname{IgG}$ and serious infections occurred, leading to early termination of the trial after only six patients had been enrolled. As a result, no efficacy evaluation took place. These results are likely to be explained by factors discussed later in this article.

\section{Atacicept: concerns about tolerability and safety}

Concern has been raised that patients treated with atacicept might have a greater potential for developing serious adverse events (AEs) related to immunosuppression and consequent serious infections. ${ }^{7}$

In the Phase 1 studies conducted in healthy volunteers, in patients with moderate to severe RA and in patients with SLE, atacicept was found to be well tolerated. There was no significant increase in the frequency of infection-related events between patients who received atacicept and those who received placebo. . $3,55,60,61$

In AUGUST I although the overall AEs, AEs leading to discontinuation and serious AEs (SAEs) were more frequent in the atacicept groups, there were no dose-dependent trends in AEs. ${ }^{56}$ There was no difference in terms of infectionrelated AEs between placebo and atacicept groups. In two patients randomized to the $150 \mathrm{mg}$ group, three serious infection-related AEs were reported. One patient from the atacicept $25 \mathrm{mg}$ group died from postoperative sepsis and one patient who was receiving atacicept $75 \mathrm{mg}$ died of sudden cardiac arrest, but the latter was considered unlikely to be related to treatment.

In AUGUST II patients treated with atacicept had a higher frequency of AEs than those receiving placebo. ${ }^{57}$ Patients treated with atacicept $150 \mathrm{mg}$ had a slightly higher incidence of infection-related AEs compared to those receiving placebo (35\% versus $29 \%$ ). While no serious infections were reported in patients randomized to atacicept, one patient treated with placebo had cellulitis and three cases of SAEs were reported in the adalimumab group (two cases of pneumonia and one case of disseminated tuberculosis). Five patients, each previously randomized to atacicept, developed a tumor (four 
benign tumors and one breast cancer). One patient treated with atacicept died of sudden cardiac arrest. This patient had a history of diabetes and hypertension.

In both AUGUST I and AUGUST II, median IgG levels remained above the lower limit of normal throughout the study, and no patient discontinued atacicept because of IgG levels below $3 \mathrm{~g} / \mathrm{L} .^{56,57}$

In the study designed to assess whether atacicept could prevent flares in patients with previous lupus disease activity, two fatal infections occurred in the $150 \mathrm{mg}$ arm, resulting in the premature termination of this arm. However, most treatment related AEs were mild or moderate. In patients receiving atacicept, median IgG levels remained above the lower limit of normal throughout the study. ${ }^{3}$ Only two patients reached IgG levels below $3 \mathrm{~g} / \mathrm{L}$ and, even in those, no serious infections were reported. Of note, the infections in the two patients who died were not associated with hypergammaglobulinemia. The incidence of infection was not influenced by the degree of decline in IgG or IgM levels. Moreover, although the rate of total infections and serious infections was slightly higher with atacicept $150 \mathrm{mg}$, in neither atacicept arm was the risk of serious infection statistically significantly increased compared with the placebo arm. Also, the overall similarity in AEs between the three arms of the study, including SAEs, was reassuring. The rate of serious infections in the BLISS 76 study $^{44}$ (7\% and $7.3 \%$ in the 1 and $10 \mathrm{mg} / \mathrm{kg}$ arms, respectively) was virtually identical to the $6.9 \%$ observed in the atacicept $150 \mathrm{mg}$ arm of the Phase II/III study. In the EXPLORER trial ${ }^{12}$ of rituximab in patients with non-renal SLE, the rate of serious infections was even higher $(17 \%$ in the placebo group and $9.5 \%$ in the rituximab group), perhaps reflecting the more intensive use of steroids and immunosuppressives. These results suggest that the underlying disease, steroid therapy, and delays in diagnosis and treatment may have been contributing factors to the deaths observed in the Phase II/III atacicept study. ${ }^{3}$

The Phase II/III trial of atacicept in patients with LN was terminated early due to safety concerns. ${ }^{4}$ By the first day, serum IgG levels had substantially declined in patients then randomized to atacicept compared with placebo and IgG decline continued following initiation of atacicept. Among the atacicept-treated patients, three developed serum IgG below the discontinuation threshold of $3 \mathrm{~g} / \mathrm{L}$, two of whom developed serious pneumonia. The results can be, at least partially explained by factors other than atacicept itself. First, the decline in IgG began 2 weeks before the initiation of atacicept, when MMF and high-dose steroids were initiated. Of note, a decline in IgG synthesis was reported in a patient with SLE after high-dose steroids therapy. ${ }^{63}$ Furthermore, hypergammaglobulinemia has been reported in approximately half of renal transplant patients who received MMF and steroids and an increased risk of infection has been observed in other LN studies during the induction phase with MMF and high-dose CS. ${ }^{64-66}$ These results raise the possibility that severe hypogammaglobulinaemia might be responsible for this increased risk of infection. Secondly, by chance, the patients who were subsequently randomized to atacicept had higher proteinuria, at screening and through to day 14 , than those given placebo. They also had urine protein:creatinine ratios $\geq 3.0 \mathrm{mg} / \mathrm{mg}$ at screening. The extent of $\mathrm{IgG}$ decline (up to $57 \%$ in the 2 weeks before atacicept initiation) suggests that an abnormally elevated excretion of IgG in the context of heavy proteinuria and a decrease in $\mathrm{IgG}$ production secondary to MMF and steroids administration may have played a role. The pharmacokinetics of atacicept may also have been influenced by the high levels of proteinuria. However, the true impact of higher proteinuria and reduced glomerular filtration rate on atacicept secretion is unknown as baseline glomerular filtration rate was lower in the patients who received atacicept. The two patients who developed serious pneumonia had lymphopenia and low $\mathrm{CD}^{+}$counts. In fact, lymphocyte counts were low in all patients at baseline, before MMF administration, which could be explained by active lupus, as well as CS use. Overall it is evident that the risk of infection associated with the use of atacicept (in those patients) is not different from that of other biologic agents notably belimumab and rituximab.

\section{Conclusion}

Identifying new therapies with improved risk to benefit ratio is needed, particularly for patients with SLE who do not respond satisfactorily to conventional therapies (steroids and immunosuppressives). Several studies suggest that BLyS and APRIL play an important role in the pathogenesis of autoimmune diseases, including SLE. Moreover, it has been reported that neutralizing both BLyS and APRIL might be more effective than neutralizing BLyS alone. Hence, atacicept emerges as an attractive therapeutic option. Its biological activity has been demonstrated in numerous studies performed in animals as well as in patients with autoimmune diseases, including SLE. In the study designed to evaluate atacicept in the prevention of flares in patients with SLE, the results with the dose of $150 \mathrm{mg}$ were also encouraging. With regard to safety concerns, careful interpretation of the data is reassuring. The results of the atacicept trial in patients with LN suggest that minimizing the use of concurrent immunosuppressive medications, notably mycophenolate, may diminish the risk 
of infection while improving clinical response. Atacicept may provide an added value in treating autoimmune conditions such as SLE and further investigations are warranted to establish its efficacy and safety in these diseases.

\section{Acknowledgment}

We thank Dr Claudia Pena Rossi for her constructive criticism of the manuscript.

\section{Disclosure}

Professor David Isenberg has in the past acted as a consultant for Merck Serono. The honoraria provided were passed onto a local arthritis charity. The remaining authors have declared no conflicts of interest.

\section{References}

1. Harvey PR, Gordon C. B-Cell Targeted Therapies in Systemic Lupus Erythematosus, Successes and Challenges. Bio Drugs. 2013;27(2): $85-95$.

2. Leandro MJ, Cambridge G, Edwards JC, Ehrenstein MR, Isenberg DA. B cell depletion in the treatment of patients with systemic lupus erythematosus: a longitudinal analysis of 24 patients. Rheumatology (Oxford). 2005;44(12):1542-1545.

3. Isenberg D, Gordon C, Licu D, Copt S, Rossi CP, Wofsy D. Efficacy and safety of atacicept for prevention of flares in patients with moderate-tosevere systemic lupus erythematosus (SLE): 52-week data (APRIL-SLE randomised trial). Ann Rheum Dis. Epub 2014 Jun 20.

4. Ginzler EM, Wax S, Rajeswaran A, et al. Atacicept in combination with MMF and corticosteroids in lupus nephritis: results of a prematurely terminated trial. Arthritis Res Ther. 2012;14(1):R33.

5. Kamal A, Khamashta M. The efficacy of novel B cell biologics as the future of SLE treatment: A review. Autoimmun Rev. 2014;13(11): 1094-1101.

6. Nestorov I, Papasouliotis O, Pena Rossi C, Munafo A. Pharmacokinetics and Immunoglobulin Response of Subcutaneous and Intravenous Atacicept in Patients With Systemic Lupus Erythematosus. J Pharm Sci. 2010;99(1):524-538.

7. Stohl W. Biologic Differences Between Various Inhibitors of the BLyS/BAFF Pathway: Should We Expect Differences Between Belimumab and Other Inhibitors in Development? Curr Rheumatol Rep. 2012;14(4):303-309.

8. Stohl W. Therapeutic targeting of the BAFF/APRIL axis in systemic lupus erythematosus. Expert Opin Ther Targets. 2014;18(4):473-489.

9. Richez C, Truchetet ME, Schaeverbeke T, Bannwarth B. Atacicept as an investigated therapy for rheumatoid arthritis. Expert Opin Investig Drugs. 2014;23(9):1285-1294.

10. Krumbholz M, Meinl E. B cells in MS and NMO: pathogenesis and therapy. Semin Immunopathol. 2014;36(3):339-350.

11. Furtado J, Isenberg DA. B cell elimination in systemic lupus erythematosus. Clin Immunol. 2013;146(2):90-103.

12. Merrill JT, Neuelt CM, Wallace DJ, et al. Efficacy and safety of rituximab in moderately-to-severely active systemic lupus erythematosus: the randomized, double-blind, phase II/III systemic lupus erythematosus evaluation of rituximab trial. Arthritis Rheum. 2010;62(1):222-233.

13. Rovin BH, Furie R, Latinis K, et al. Efficacy and safety of rituximab in patients with active proliferative lupus nephritis: the Lupus Nephritis Assessment with Rituximab study. Arthritis Rheum. 2012; 64(4):1215-1226.

14. Dillon SR, Gross JA, Ansell SM, Novak AJ. An April to remember: novel TNF ligands as therapeutic targets. Nat Rev Drug Discov. 2006;5(3):235-246.
15. Castigli E, Wilson SA, Scott S, et al. TACI and BAFF-R mediate isotype switching in B cells. J Exp Med. 2005;201(1):35-39.

16. Stohl W, Scholz JL, Cancro MP. Targeting BLyS in rheumatic disease: the sometimes-bumpy road from bench to bedside. Curr Opin Rheumatol. 2011;23(3):305-310.

17. Stein JV, López-Fraga M, Elustondo FA, et al. APRIL modulates B and T cell immunity. J Clin Invest. 2002;109(12):1587-1598.

18. Schneider P, MacKay F, Steiner V, et al. BAFF, a novel ligand of the tumor necrosis factor (TNF) family, stimulates B-cell growth. $J$ Exp Med. 2011;189(11):1747-1756.

19. Yang M, Hase H, Legarda-Addison D, Varughese L, Seed B, Ting AT. $\mathrm{B}$ cell maturation antigen, the receptor for a proliferation-inducing ligand and B cell-activating factor of the TNF family, induces antigen presentation in B cells. J Immunol. 2005;175(5):2814-2824.

20. Yu G, Boone T, Delaney J, et al. APRIL and TALL-I and receptors BCMA and TACI: system for regulating humoral immunity. Nature Immunol. 2000;1(3):252-256.

21. Stein JV, López-Fraga M, Elustondo FA, et al. APRIL modulates B and T cell immunity. $J$ Clin Invest. 2002;109(12):1587-1598.

22. Planelles L, Carvalho-Pinto CE, Hardenberg G, et al. APRIL promotes B-1 cell-associated neoplasm. Cancer Cell. 2004;6(4):399-408.

23. Jacob CO, Pricop L, Putterman C, et al. Paucity of clinical disease despite serological autoimmunity and kidney pathology in lupus prone New Zealand Mixed 2328 mice deficient in BAFF. J Immunol. 2006;177(4):2671-2680.

24. Ramanujam M, Wang X, Huang W, et al. Mechanism of action of transmembrane activator and calcium modulator ligand interactor-Ig in murine systemic lupus erythematosus. $J$ Immunol. 2004;173(5): 3524-3534.

25. Gross JA, Johnston J, Mudri S, et al. TACI and BCMA are receptors for a TNF homologue implicated in B-cell autoimmune disease. Nature. 2000; 404(6781):995-999.

26. Mackay F, Woodcock SA, Lawton P, et al. Mice transgenic for BAFF develop lymphocytic disorders along with autoimmune manifestations. J Exp Med. 1999;190(11):1697-1710.

27. Khare SD, Sarosi I, Xia XZ, et al. Severe B cell hyperplasia and autoimmune disease in TALL-1 transgenic mice. Proc Natl Acad Sci U S A. 2000;97(7):3370-3375.

28. Stohl W, Xu D, Kim KS, et al. BAFF overexpression and accelerated glomerular disease in mice with an incomplete genetic predisposition to systemic lupus erythematosus. Arthritis Rheum. 2005;52(7):2080-2091.

29. Morel J, Hahne M. To target or not to target APRIL in systemic lupus erythematosus: that is the question! Arthritis Res Ther. 2013;15(1): $107-108$.

30. Varfolomeev E, Kischkel F, Martin F, et al. APRIL-deficient mice have normal immune system development. Mol Cell Biol. 2004; 24(3):997-1006.

31. Castigli E, Scott S, Dedeoglu F, et al. Impaired IgA class switching in APRIL-deficient mice. Proc Natl Acad Sci U S A. 2004;101(11): 3903-3908.

32. Jacob CO, Guo S, Jacob N, et al. Dispensability of APRIL to the development of systemic lupus erythematosus in NZM 2328 mice. Arthritis Rheum. 2012;64(5):1610-1619.

33. Munafo A, Priestley A, Nestorov I, Visich J, Rogge M. Safety, pharmacokinetics and pharmacodynamics of atacicept in healthy volunteers. Eur J Clin Pharmacol. 2007;63(7):647-656.

34. Kappos L, Hartung HP, Freedman MS, et al. Atacicept in multiple sclerosis (ATAMS): a randomised, placebo-controlled, double-blind, phase 2 trial. Lancet Neurol. 2014;13(4):353-363.

35. Kofler DM, Gawlik BB, Elter T, Gianella-Borradori A, Wendtner CM, Hallek M. Phase $1 \mathrm{~b}$ trial of atacicept, a recombinant protein binding BLyS and APRIL, in patients with chronic lymphocytic leukemia. Leukemia. 2012;26(4):841-844. 
36. Koyama T, Tsukamoto H, Miyagi Y, et al. Raised serum APRIL levels in patients with systemic lupus erythematosus. Ann Rheum Dis. 2005;64(7):1065-1067.

37. Stohl W, Metyas S, Tan SM, et al. Inverse association between circulating APRIL levels and serological and clinical disease activity in patients with systemic lupus erythematosus. Ann Rheum Dis. 2004;63(9):1096-1103.

38. Sutherland AP, Ng LG, Fletcher CA, et al. BAFF augments certain Th1-associated inflammatory responses. J Immunol. 2005; 174(9):5537-5544.

39. Zhou X, Xia Z, Lan Q, et al. BAFF promotes Th17 cells and aggravates experimental autoimmune encephalomyelitis. PLoS One. 2011;6(8):e23629.

40. Fairfax K, Mackay IR, Mackay F. BAFF/BLyS Inhibitors: A New Prospect for Treatment of Systemic Lupus Erythematosus. IUBMB Life. 2012;64(7):595-602.

41. Wallace DJ, Stohl W, Furie RA, et al. A phase II, randomized, double-blind, placebo-controlled, doseranging study of belimumab in patients with active systemic lupus erythematosus. Arthritis Rheum. 2009;61(9):1168-1178.

42. Furie RA, Petri MA, Wallace DJ, et al. Novel evidence-based systemic lupus erythematosus responder index. Arthritis Rheum. 2009;61(9):1143-1151.

43. Navarra SV, Guzmán RM, Gallacher AE, et al. Efficacy and safety of belimumab in patients with active systemic lupus erythematosus: a randomized placebo-controlled, phase 3 trial. Lancet. 2011; 377(9767):721-731.

44. Furie R, Petri M, Zamani O, et al. A phase III, randomized, placebocontrolled study of belimumab, a monoclonal antibody that inhibits B lymphocyte stimulator, in patients with systemic lupus erythematosus. Arthritis Rheum. 2011;63(12):3918-3930.

45. Manzi S, Sánchez-Guerrero J, Merrill JT, et al. BLISS-52 and BLISS-76 Study Groups: Effects of belimumab, a B lymphocyte stimulatorspecific inhibitor, on disease activity across multiple organ domains in patients with systemic lupus erythematosus: combined results from two phase III trials. Ann Rheum Dis. 2012;71(11):1833-1838.

46. Dooley MA, Houssiau F, Aranow C, et al. Effect of belimumab treatment on renal outcomes: results from the phase 3 belimumab clinical trials in patients with SLE. Lupus. 2013;22(1):63-72.

47. Van Vollenhoven RF, Petri MA, Cervera R, et al. Belimumab in the treatment of systemic lupus erythematosus: high disease activity predictors of response. Ann Rheum Dis. 2012;71(8):1343-1349.

48. Vincent FB, Saulep-Easton D, Figgett WA, Fairfax KA, Mackay F. The BAFF/APRIL system: Emerging functions beyond B cell biology and Autoimmunity. Cytokine Growth Factor Rev. 2013;24(3):203-215.

49. Ramanujam M, Wang X, Huang W, et al. Similarities and differences between selective and nonselective BAFF blockade in murine SLE. $J$ Clin Invest. 2006;116(3):724-734.

50. Gross JA, Dillon SR, Mudri S, et al. TACI-Ig neutralizes molecules critical for B cell development and autoimmune disease: impaired B cell maturation in mice lacking BLyS. Immunity. 2001;15(2):289-302.

51. Wang H, Marsters SA, Baker T, et al. TACI-ligand interactions are required for $\mathrm{T}$ cell activation and collagen-induced arthritis in mice. Nat Immunol. 2001;2(7):632-637.

52. Carbonatto M, Yu P, Bertolino M, et al. Nonclinical safety, pharmacokinetics, and pharmacodynamics of atacicept. Toxicol Sci. 2008;105(1):200-210.
53. Krumbholz M, Derfuss T, Hohlfeld R, Meinl E. B cells and antibodies in multiple sclerosis pathogenesis and therapy. Nat Rev Neurol. 2012;8(11):613-623.

54. Kretzschmar B, Hein K, Moinfar Z, et al. Treatment with atacicept enhances neuronal cell death in a rat model of optic neuritis. J Neuroimmunol. 2014;268(1-2):58-63.

55. Tak PP, Thurlings RM, Rossier C, et al. Atacicept in Patients With Rheumatoid Arthritis: Results of a Multicenter, Phase Ib, Double-Blind, Placebo-Controlled, Dose-Escalating, Single- and Repeated-Dose Study. Arthritis Rheum. 2008;58(1):61-72.

56. Genovese MC, Kinnman N, de La Bourdonnaye G, Pena Rossi C, Tak PP. Atacicept in patients with rheumatoid arthritis and an inadequate response to tumor necrosis factor antagonist therapy: results of a phase II, randomized, placebo-controlled, dose-finding trial. Arthritis Rheum. 2011;63(7):1793-1803.

57. Van Vollenhoven RF, Kinnman N, Vincent E, Wax S, Bathon J. Atacicept in patients with rheumatoid arthritis and an inadequate response to methotrexate: results of a phase II, randomized, placebocontrolled trial. Arthritis Rheum. 2011;63(7):1782-1792.

58. Ansell SM, Witzig TE, Inwards DJ, et al. Phase I clinical study of atacicept in patients with relapsed and refractory Bcell non-Hodgkin's lymphoma. Clin Cancer Res. 2008;14(4):1105-1110.

59. Rossi JF, Moreaux J, Hose D, et al. Atacicept in relapsed/refractory multiple myeloma or active Waldenstrom's macroglobulinemia: a phase I study. Br J Cancer. 2009;101(7):1051-1058.

60. Dall'Era M, Chakravarty E, Wallace D, et al. Reduced B lymphocyte and immunoglobulin levels after atacicept treatment in patients with systemic lupus erythematosus, results of a multicenter, phase $\mathrm{Ib}$, double-blind, placebo-controlled, dose-escalating trial. Arthritis Rheum. 2007;56(12):4142-4150.

61. Pena-Rossi C, Nasonov E, Stanislav M, et al. An exploratory doseescalating study investigating the safety, tolerability, pharmacokinetics and pharmacodynamics of intravenous atacicept in patients with systemic lupus Erythematosus. Lupus. 2009;18(6):547-555.

62. Treamtrakanpon W, Tantivitayakul P, Benjachat T, et al. APRIL, a proliferation inducing ligand, as a potential marker of lupus nephritis. Arthritis Res Ther. 2012;14(6):R252.

63. McMillan R, Longmire R, Yelenosky R. The effect of corticosteroids on human IgG synthesis. J Immunol. 1976;116(6):1592-1595.

64. Broeders EN, Wissing KM, Hazzan M, et al. Evolution of immunoglobulin and mannose binding protein levels after renal transplantation: association with infectious complications. Transpl Int. 2008; 21(1):57-64.

65. Tucci M, Stucci S, Strippoli S, Silvestris F. Cytokine overproduction, T-cell activation, and defective T-regulatory functions promote nephritis in systemic lupus erythematosus. J Biomed Biotechnol. 2010;2010:457146.

66. Roschke V, Sosnovtseva S, Ward CD, et al. BLyS and APRIL form biologically active heterotrimers that are expressed in patients with systemic immune-based rheumatic diseases. J Immunol. $2002 ; 169(8): 4314-4321$.
Drug Design, Development and Therapy

\section{Publish your work in this journal}

Drug Design, Development and Therapy is an international, peerreviewed open-access journal that spans the spectrum of drug design and development through to clinical applications. Clinical outcomes, patient safety, and programs for the development and effective, safe, and sustained use of medicines are a feature of the journal, which

\section{Dovepress}

has also been accepted for indexing on PubMed Central. The manuscript management system is completely online and includes a very quick and fair peer-review system, which is all easy to use. Visit http://www.dovepress.com/testimonials.php to read real quotes from published authors. 